\title{
Amplification of Light in Absorbing Media: Stimulated Thermal Rayleigh Scattering
}

\author{
W. ROTHER, H. MEYeR, and W. KAISER \\ Physik-Department der Technischen Hochschule München \\ (Z. Naturforsch. 25 a, 1136-1143 [1970] ; received 4 Mai 1970)
}

\begin{abstract}
Using well defined input pulses the amplification by stimulated thermal Rayleigh scattering was investigated experimentally as a function of time (within the pulse), of absorption coefficient, and of interaction length. The frequency difference between the two interacting light pulses was varied with an accuracy better than $\Delta v / v=10^{-8}$. Amplification was measured in the backward direction and at several degrees from the forward direction. The various experimental observations are in good agreement with theory. The possibility of determining material parameters in this amplifier system is discussed.
\end{abstract}

\section{A) Introduction}

In recent years stimulated light scattering in absorbing media has received considerable attention. Most previous publications were concerned with the steady state situation, which presents less difficulty to a theoretical treatment. The short light pulses used experimentally $\left(\cong 10^{-8} \mathrm{sec}\right)$ and the longer time constants of the material excitation suggested the necessity of transient investigations. In the preceding paper ${ }^{1}$, referred to as I, a transient theory of stimulated light scattering in absorbing media is developed under the assumption of small signal and small amplification. These results are well suited for an analysis of measurements obtained in an amplifier system. In this paper a quantitative comparison between theory and experiment will be presented.

It has been shown theoretically that in addition to stimulated Brillouin scattering (scattering by propagating density fluctuations or phonons) and stimulated Rayleigh scattering (scattering by nonpropagating temperature fluctuations) new stimulated scattering processes are expected in absorbing me$\mathrm{dia}^{2}$. Temperature fluctuations generated in absorbing liquids or gases give rise to density fluctuations which, in turn, are responsible for light scattering. Stimulated thermal scattering is observed within the frequency range of the Rayleigh and the Brillouin line. The frequency difference between the pump and scattered signal, determines whether we

Sonderdruckanforderungen an W. KAISER, Physik-Department der Techn. Hochschule München, D-8000 München 2, Arcisstraße 21

1 W. Rother, preceding paper, Z. Naturforsch. 25 a, 1120 [1970].

2 R. M. Herman and M. A. Gray, Phys. Rev. Letters 19, 824 [1967]. investigate stimulated thermal Rayleigh scattering (STRS) or stimulated thermal Brillouin scattering (STBS).

The first theoretical investigation of the steady state case of stimulated thermal scattering processes was made by Herman and GraY in $1967^{2}$. Using a generator-amplifier system PoHL et al. ${ }^{3}$ investigated the frequency and time dependence of stimulated thermal Brillouin scattering and found excellent agreement with the steady state theory. This fact was expected since time resolved measurements clearly indicated that the steady state was attained in these experiments.

Stimulated thermal Rayleigh scattering has been studied experimentally by a number of authors in a travelling wave system ${ }^{4}$. The scattered signal was amplified from noise to the intensity level of the incident laser source and investigated with time integrating photodetectors and Fabry-Perot interferometers. Discrepancies were noticed between the steady state theory and experimental observations.

In this paper experimental investigations of stimulated thermal Rayleigh scattering are presented in an amplifier system of high time and frequency resolution. For backward scattering $\left(180^{\circ}\right)$ the Rayleigh line width is approximately $\Gamma_{\mathrm{R}} / 2 \pi=20 \mathrm{MHz}$ for many liquids; i. e. the relaxation time of the corresponding density fluctuations is $\left(\Gamma_{\mathrm{R}}\right)^{-1}=10 \mathrm{nsec}$. This value has to be compared with the half width of our approximately Gaussian laser pulse of

${ }^{3}$ D. Pohl, I. Reinhold, and W. Kaiser, Phys. Rev. Letters 20, 1141 [1968].

${ }^{4}$ D. H. Rank, C. W. Cho, N. D. Foltz, and T. A. Wiggins, Phys. Rev. Letters 19, 828 [1967]. - T. A. Wiggins, C. W. Cho, D. R. Dietz, and N. D. Foltz, Phys. Rev. Letters 20, 831 [1968]. - C. W. Cho, N. D. Foltz, D. H. Rank, and T. A. Wiggins, Phys. Rev. 175, 271 [1968]. - F. Gires, C. R. Acad. Sci. Paris 266 B, 596 [1968]. 
$t_{\mathrm{h}}=15$ nsec. It has been shown in I that light amplification by STRS is strongly transient under these conditions and far from the steady state situation. The most striking theoretical prediction is the transient amplification for the case of $\omega=0$, i. e. when the pump and signal have the same frequency. Steady state calculations predict zero gain at $\omega=0$. While the theory of $\mathrm{I}$ is not readily extended to the case of high gain and large signals, the transient nature of STRS should also be considered there. Preliminary experimental observations with high time resolution support this prediction.

In Section B theoretical results of I are repeated as far as they are relevant to the interpretation of our experimental results. Our experimental system and various experimental investigations are discussed in Sections C and D respectively. Concluding remarks and summary are given in Section E.

\section{B) Theoretical Remarks}

Here theoretical results derived in I are summarized; they are extended to take into account the intensity profile within the incident laser beam. In this way a quantitative comparison with our experimental observations is readily possible.

The amplification $V_{\mathrm{R}}$ of an input signal $I_{1}(0, \tau)$ and the time dependent gain factor $g_{\mathrm{R}}^{a}(L, \omega, \tau)$ have been calculated for a variety of conditions in I (see Figs. 2, 3, 4, and 6 a). They are defined and related as follows:

$$
\begin{gathered}
V_{\mathrm{R}}=I_{1}(L, \tau) / I_{1}(0, \tau)-1 ; \\
V_{\mathrm{R}}=\exp \left\{g_{\mathrm{R}}^{\mathrm{a}}(L, \omega, \tau) H^{2}(\tau) L / \widehat{g_{\mathrm{R}}^{\mathrm{a}}}\right\}-1 ;
\end{gathered}
$$

here $\tau=\Gamma_{\mathrm{R}} t / 2$ is a dimensionless time with

$$
\Gamma_{\mathrm{R}}=2 \lambda k^{2} / \varrho_{0} c_{p}
$$

the Rayleigh line width of the medium; $\lambda$ the thermal conductivity, $\varrho_{0}$ the density and $c_{p}$ the specific heat are material parameters. The $k$-vector of the scattering density wave depends upon the angle $\Theta$ between the incident and the scattered beam.

$$
k=2 k_{0} \sin (\Theta / 2)
$$

where $k_{0}=2 \pi / \lambda_{0}=\omega_{0} n / c$ is the $k$-vector of the pump wave. On account of Eq. (4) the relaxation time $1 / \Gamma_{\mathrm{R}}$ is quite large in the near forward direction, e. g. $1 / \Gamma_{\mathrm{R}} \approx 400 \mathrm{nsec}$ is calculated for $\Theta=10^{\circ}$ in $\mathrm{CCl}_{4}$.

The quantity $L=\hat{g}_{\mathrm{R}}^{\mathrm{a}} I_{0} z$ is a measure of the gain; $I_{0}$ is the peak intensity of the pump pulse and $\hat{g}_{\mathrm{R}}^{\mathrm{a}}$ represents the maximum steady state gain factor of STRS:

$$
\widehat{g_{\mathrm{R}}^{\mathrm{a}}}=\gamma^{\mathrm{e}} \gamma^{\mathrm{a}} \omega_{1} k / 2 n^{2} c^{2} v \varrho_{0} \Gamma_{\mathrm{R}} .
$$

The electro-optic coupling constant $\gamma^{\mathrm{e}}$ and the thermo-optic coupling constant $\gamma^{\mathrm{a}}$ are defined as follows :

$$
\begin{aligned}
& \gamma^{\mathrm{e}}=\varrho_{0}(\partial \varepsilon / \partial \varrho)_{T}, \\
& \gamma^{\mathrm{a}}=v c^{2} \alpha \beta / c_{p} \omega_{0} \sin (\Theta / 2) .
\end{aligned}
$$

In liquids we can write, to a good approximation, $\gamma^{\mathrm{e}}=\left(n^{2}-1\right)\left(n^{2}+2\right) / 3 ; \alpha$ is the absorption coefficient, $\beta$ the thermal expansion coefficient, $v$ the acoustic velocity and $n$ the index of refraction of the medium. $\omega=\omega_{0}-\omega_{1}$ represents the frequency difference between pump and signal wave. It is assumed in the computer calculations that the pump and signal pulse have the same Gaussian time dependence

$$
I_{0}=\hat{I_{0}} H^{2}(\tau) \quad \text { and } \quad I_{1}=\hat{I_{1}} H^{2}(\tau) .
$$

For small signals and small amplification the following expression was derived for $V_{\mathrm{R}}$ :

$$
V_{\mathrm{R}}=C_{1}(\tau, \omega) L+C_{2}(\tau, \omega) L^{2} .
$$

$C_{1}$ and $C_{2}$ are complicated functions of $\omega$, the dimensionless time and the half width of the Gaussian pulse $t_{\mathrm{h}}$. The situation is substantially simplified in the important case of equal frequencies for pump and signal wave. It can be shown quite generally that $C_{1} \equiv 0$ and $C_{2} \neq 0$ for $\omega=0$. Experimentally it is convenient to measure the amplification of the incident signal pulse at the time when the pump pulse has its maximum intensity. In this way accurate data are obtained. Computer calculations were performed with the origin of the time scale set at the maximum of the Gaussian pump pulse. In Fig. 1 calculated values of $C_{2}(\tau=0, \omega=0)$ are plotted versus the normalized half width $\tau_{\mathrm{h}}$ of the pump pulse. It is readily seen from the Figure that $C_{2}$ is well approximated for $t_{\mathrm{h}}<\mathrm{l} / 2 \Gamma_{\mathrm{R}}$ by the equation:

$$
C_{2}(\tau=0, \omega=0)=3.2 \times 10^{-2}\left(t_{\mathrm{h}} \Gamma_{\mathrm{R}}\right)^{2}
$$

i. e. $C_{2}$ depends quadratically upon $t_{\mathrm{h}} \Gamma_{\mathrm{R}}$ when the electromagnetic excitation is shorter than the relaxation time of the medium. Since $L^{2}$ is proportional to $\left(\Gamma_{\mathrm{R}}\right)^{-2}$, we obtain the interesting but expected result, that the strongly transient amplification is independent of $\Gamma_{\mathrm{R}}$. Equation (10) holds well for scattering in the forward direction where $t_{\mathrm{h}} \ll 1 / 2 \Gamma_{\mathrm{R}}$ (see Sect. D 3). In the backward direction, where 
$t_{\mathrm{h}} \cong 1 / 2 \Gamma_{\mathrm{R}}$, Fig. 1 has to be used for each product $t_{\mathrm{h}} \Gamma_{\mathrm{R}}$ (see Sect. D lb).

Equations (1) and (2) contain light intensities for the signal and pump beam. Experimentally

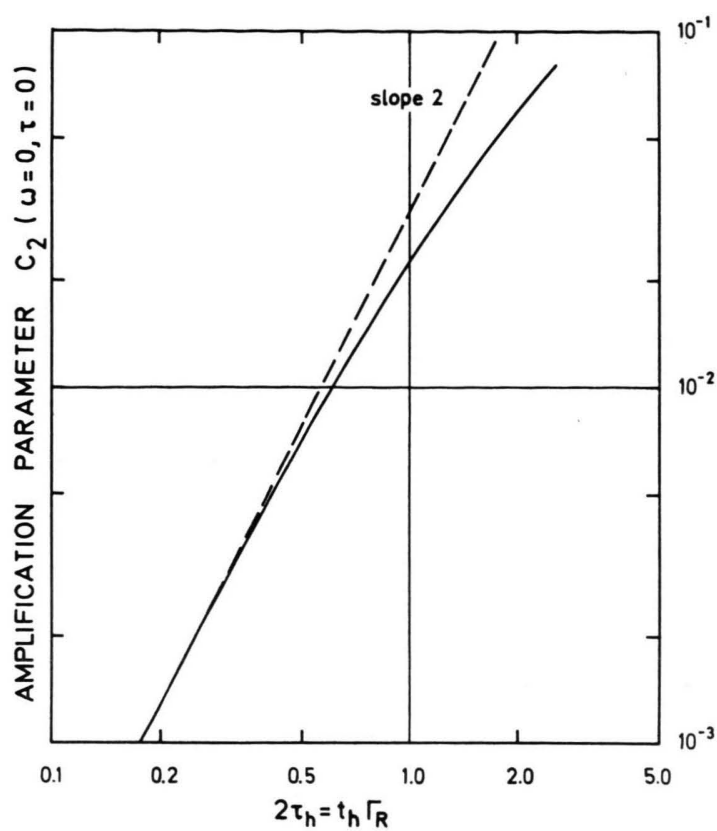

Fig. 1. Amplification parameter $C_{2}$ for the peak of the light pulse and for $\omega=0$ versus $t_{\mathrm{h}} \Gamma_{\mathrm{R}}$ (halfwidth of Gaussian pulse $\times$ Rayleigh line width).

power values are measured with our fast photocells. Our laser operated in the $\mathrm{TEM}_{00}$ mode which is known to have a Gaussian intensity profile with the spot size $W$.

$$
I(\tau, r)=I(\tau) \exp \left\{-2 r^{2} / W^{2}\right\} .
$$

In our experimental system the pump and signal pulse have the same radial profile of Eq. (11). For small amplification we can readily integrate over the cross section of the beam and obtain:

$$
G=C_{1}(\tau, \omega) U+4 C_{2}(\tau, \omega) U^{2} / 3
$$

where $G$ is the power amplification

$$
G=P_{\mathrm{a}} e^{+a l} / P_{\mathrm{i}}-1
$$

of the output and input pulse, $P_{\mathrm{a}}(t)$ and $P_{\mathrm{i}}(t)$ respectively. The abbreviation

$$
U=\hat{g}_{\mathrm{R}}^{\mathrm{a}} \hat{P}_{0} l / W^{2} \pi
$$

is introduced where $\hat{P}_{0}$ represents the peak power of the pump pulse $\left(\hat{P}_{0}=\int \hat{I}_{0} \mathrm{~d} f\right)$ and $l$ the interaction length. The factors $C_{1}(\tau, \omega)$ and $C_{2}(\tau, \omega)$ have to be calculated as a function of time and fre- quency for the individual pulse width $t_{\mathrm{h}}$. The following points should be noted: According to Eq. (12), the amplification $G$ for $\omega=0$ is proportional to $U^{2}$, i. e. $G$ is expected to be proportional to $\alpha^{2}$ and $l^{2}$. Experiments discussed in Section D confirm this prediction.

\section{C) Experimental}

The following experiments were performed with a single mode, giant pulse, laser. A ruby crystal, $7.5 \mathrm{~cm}$ in length, was mounted in a spherical resonator and passively $Q$-switched with cryptocyanine (in methanol) . Resonant reflectors and an adjustable diaphragm within the resonator selected the fundamental $\mathrm{TEM}_{00}$ resonant mode of the system. The time dependence of the pulse was to a good approximation Gaussian with a half width of $t_{\mathrm{h}}=14$ nsec.

It has been shown by a number of authors that the emission frequency of the laser drifts during the emission of the pulse ${ }^{5}$. The laser used in this investigation had a measured linear frequency drift of $10 \mathrm{MHz}$ per nsec at the center of the laser pulse. This frequency drift is utilized in our gain measurements to establish a well defined frequency difference between the two interacting light pulses. Since the Rayleigh line width of many liquids is approximately $20 \mathrm{MHz}$ this technique allows investigations over the full width of the Rayleigh line.

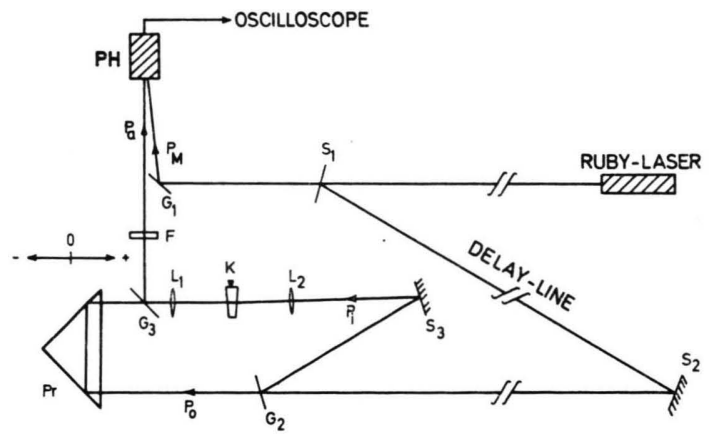

Fig. 2. Schematic of experimental system for measurements at the scattering angle of $180^{\circ}$.

In Fig. 2 the experimental system for gain measurements in the backward direction $\left(\Theta=180^{\circ}\right)$ is depicted schematically. A small amount of the incident pulse is transmitted by mirror $S_{1}$ and is directed by the glass plate $G_{1}$ into the photocell $\mathrm{PH}$. The light pulse $\mathrm{P}_{\mathrm{M}}$ is measured with a time resolution of $0.3 \mathrm{nsec}$. The major part of the input pulse is reflected by the dielectric

5 V. V. Korobkin, A. M. Leontovich, M. N. Poprava, and M. YA. Shchelev, JETP Letters 3, 194 [1966]. - D. J. Bradley, G. Magyar, and M. G. Richardson, Nature 212, 63 [1966]. - D. A. Berkley and G. J. Wolga, J. Appl. Phys. 38, 3281 [1967]. - D. Pohl, Physics Letters 26 A, 357 [1968]. 
mirror $\mathrm{S}_{1}$ with reflectivity $R=98 \%$. After travelling on an optical delay line of $16 \mathrm{~m}$ the pulse reaches the beam splitter $G_{2}$ which generates the intense pump pulse $P_{0}$ and the small input signal $P_{\mathrm{i}}$. The reflectivity of mirror $S_{3}$ is chosen in such a way that

$$
P_{\mathrm{i}} / P_{0}=2 \times 10^{-2} \text {. }
$$

A liquid cell is located close to the focal region of the two lenses $\mathrm{L}_{1}$ and $\mathrm{L}_{2}(f=30 \mathrm{~cm})$. The windows of the cell form an angle of $5^{\circ}$ to avoid oscillation within the liquid. The $\mathrm{TEM}_{00}$ mode of the laser produces at the position of the medium a spot size $W=2.1 \times 10^{-2} \mathrm{~cm}$. The prism $\operatorname{Pr}$ and mirror $S_{3}$ are adjusted that the two beams completely overlap in the cell. There is no frequency difference betwern ine interacting pulses when the two optical paths from the beam splitter $G_{2}$ to the cell are equal. Moving the prism $\mathrm{Pr}$, as indicated in Fig. 2, a frequency difference between the two pulses is introduced on account of the frequency drift mentioned above. A difference in path length of $30 \mathrm{~cm}$ results in a frequency difference of $\Delta v=10 \mathrm{MHz}$. The frequency resolution of our system is essentially determined by the length of the cell and is better than $\Delta v / v=10^{-\gamma}$.

It is readily seen from Fig. 2 that the monitor signal $P_{\mathrm{M}}$ is directlv proportional to the pump pulse $P_{\text {a }}$ and the invut signal $P_{\mathrm{i}}$. The output signal $P_{\mathrm{a}}$ is directed via the beam splitter $G_{3}$ at the same photocell $P H$; $P_{\mathrm{a}}$ arrives $55 \mathrm{nsec}$ after the pulse $P_{\mathrm{M}}$ because of the long optical delay system. Both signals are well separated on the oscilloscope trace (see Fig. 4 a). The long delay line has the additional advantage that re turning light pulses do not affect our experimental investigations. The optical components (mirrors, beam splitters and filter $\mathrm{F}$ ) are adjusted to make $P_{\mathrm{a}}$ and $P_{\mathrm{M}}=P_{\mathrm{i}}$ approximately equal when no amplification takes place (no absorption in the liquid). In this way the effect of stimulated Rayleigh scattering, amplification or attenuation, was readily visible on the oscilloscope trace.

The experimental system was modified for the investigation of light amplification at small scattering angles $\Theta$. As indicated schematically in Fig. 3, the beam splitter $\mathrm{G}_{2}$ generates the intense pump pulse $P_{0}$ and the weak input signal $P_{\mathrm{i}}$. The two beam axis cross in the center of the cell. A small rotation of mirror $S_{3}$

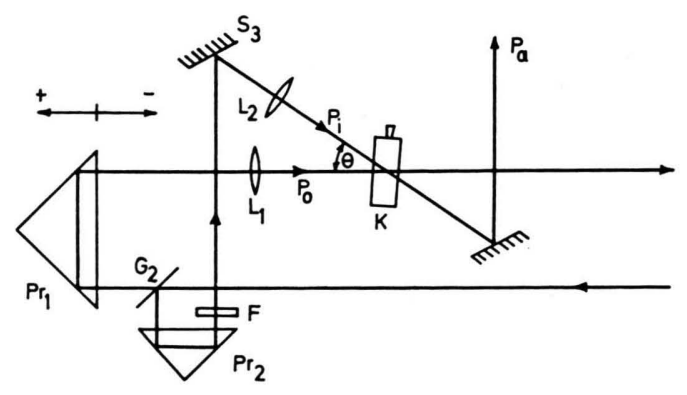

Fig. 3. Schematic of experimental system for measurements at scattering angles of several degrees. allowed a ready variation of the scattering angle $\Theta$ between $7^{\circ}$ and $16^{\circ}$. Care was taken to ensure equal path length for the two light pulses; small adjustments could be made with the help of prism $\operatorname{Pr}_{1}$. In this way the condition $\omega=0$ was maintained for different values of $\Theta$.

\section{D) Experimental Results and Discussion}

$$
\text { 1. Light Amplification for } \omega=0\left(\Theta=180^{\circ}\right)
$$

We start the discussion of the pulse amplification $G$ for $\omega=0$ in the backward direction. Three investigations are reported here: a) the dependence on time, b) on the absorption coefficient $\alpha$, and c) on the interaction length $l$. Our experiments were made using $\mathrm{CCl}_{4}$ and methanol with values for $\hat{g_{\mathrm{R}}^{\mathrm{a}}} / \alpha$ of $0.82 \mathrm{~cm}^{2} / \mathrm{MW}$ and $0.32 \mathrm{~cm}^{2} / \mathrm{MW}$ respectively. Adding small amounts of iodine in $\mathrm{CCl}_{4}$ and $\mathrm{CoCl}_{2}$ in methanol the desired values of $\alpha$ were established.
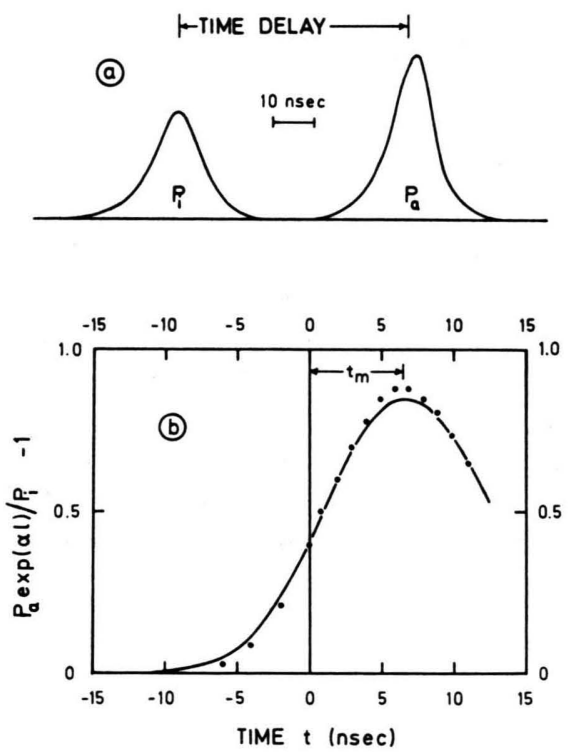

Fig. 4. a) Oscilloscope trace (redrawn) depicting the incoming signal pulse $P_{\mathrm{i}}$ and the amplified signal $P_{\mathrm{a}}$. b) Amplification versus time, experimental points and calculated curve $(\omega=0)$.

a) A typical oscilloscope trace of the input signal pulse $P_{\mathrm{i}}$ and the amplified pulse $P_{\mathrm{a}}$ is presented in Fig. 4 a. The small transient gain at the beginning of the amplification leads to an effective narrowing and a delayed maximum (@2 nsec) of the amplified pulse. A careful analysis of the pulses $P_{\mathrm{i}}$ and $P_{\mathrm{a}}$ allowed the determination of the amplification $G$ for different times during the pulse. In Fig. $4 \mathrm{~b}$, 
the solid points represent experimentally determined values of $G$ plotted as a function of time. The time $t=0$ corresponds to the time of the maximum of the pump and signal pulse. Fig. $4 \mathrm{~b}$ shows quite clearly the slowly rising amplification which reaches its maximum value several nsec $\left(t_{\mathrm{m}}\right)$ after the maximum of the electromagnetic excitation. As pointed out in I the slow build up and decay of the material excitation is responsible for this effect.

The measurements of Fig. 4 were performed under the following experimental conditions: $l=0.3 \mathrm{~cm}$, methanol with $\alpha=0.15 \mathrm{~cm}^{-1}, \hat{P}_{0}=0.22 \mathrm{MW}, W=$ $2.1 \times 10^{-2} \mathrm{~cm}$ and $t_{\mathrm{h}}=14$ nsec. For $\Gamma_{\mathrm{R}} / 2 \pi=14$ $\mathrm{MHz}$ (see below) we calculate with the help of Eq. (14) a value of $U=3.1$.

The curve depicted in Fig. $4 \mathrm{~b}$ was obtained from machine calculations of $C_{2}(\tau, 0)$ and Eq. (12). The good agreement between experimental points and the calculated curve is noteworthy. A value of $U=3.3$ was used for a best fit to our data in good agreement with the value $U=3.1$ estimated from our experimental data. To fully appreciate the accuracy of these investigations we wish to point out that $W$ and $\hat{P}_{0}$ (an absolute power value) enter critically in our calculations. The limited accuracy of our calibrated fast photodetector and the difficulty of determining $W$ very precisely are the main sources of experimental errors. Fluctuations of $G$ values of $20 \%$ between different laser pulses have been noticed.

In I, a method for the determination of $\Gamma_{\mathrm{R}}$ has been suggested using the time difference $t_{\mathrm{m}}$ between the maximum of the input pulses and the maximum

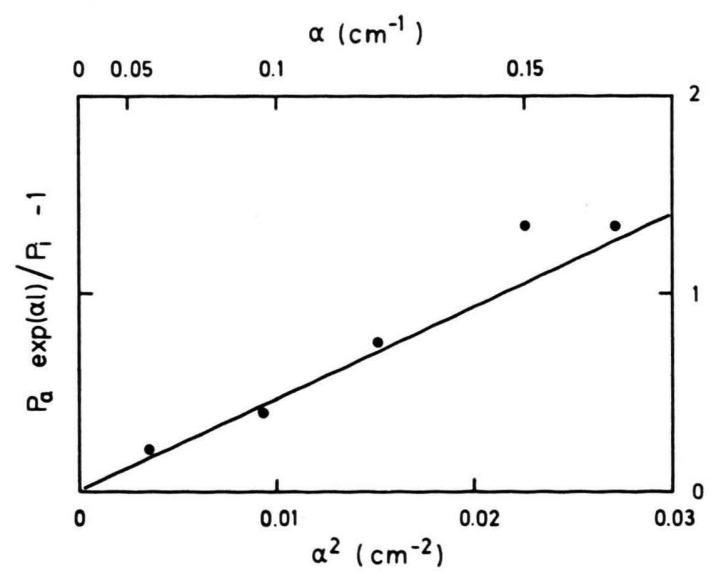

Fig. 5. Amplification at the maximum of the input pulses versus square of the absorption coefficient; experimental points and calculated line $(\omega=0)$. of the amplification $G$. Using this technique a value of $\Gamma_{\mathrm{R}} / 2 \pi=14 \mathrm{MHz}$ is deduced from Fig. $4 \mathrm{~b}$. Repeated measurements under similar conditions gave values of $\Gamma_{\mathrm{R}} / 2 \pi$ varying between $13 \mathrm{MHz}$ and $25 \mathrm{MHz}$. These numbers have to be compared with the value calculated according to Eq. (3) of $\Gamma_{\mathrm{R}} / 2 \pi$ $=20 \mathrm{MHz}$. The agreement is satisfactory considering the experimental difficulties to determine $t_{\mathrm{h}}$ and $t_{m}$ accurately and the large number of parameters entering the calculation of $\Gamma_{\mathrm{R}}$.

b) We now turn to the discussion of the dependence of the amplification $G$ on the absorption coefficient $\alpha$. Values of $\alpha$ varying between $0.06 \mathrm{~cm}^{-1}$ and $0.17 \mathrm{~cm}^{-1}$ were prepared in a cell of $0.2 \mathrm{~cm}$ length filled with $\mathrm{CCl}_{4}$. In Fig. 5 the amplification $G$ at the maximum of the input pulses $(\tau=0)$ and for $\omega=0$ is plotted as a function of $\alpha^{2}$. The different points represent values averaged over several measurements where the pulse width $t_{\mathrm{h}}$ and the pulse height $\hat{P}_{0}$ were approximately constant. Our experimental points are well accounted for by a theoretical straight line indicating the expected dependence on the square of the absorption coefficient $\alpha$. In calculating the straight line a value of $C_{2}=4.3 \times 10^{-2}$ was taken from Fig. 1 for $\Gamma_{\mathrm{R}} t_{\mathrm{h}}=1.5$; the experimental numbers of $\hat{P}_{0}$ and $W$ were listed above.
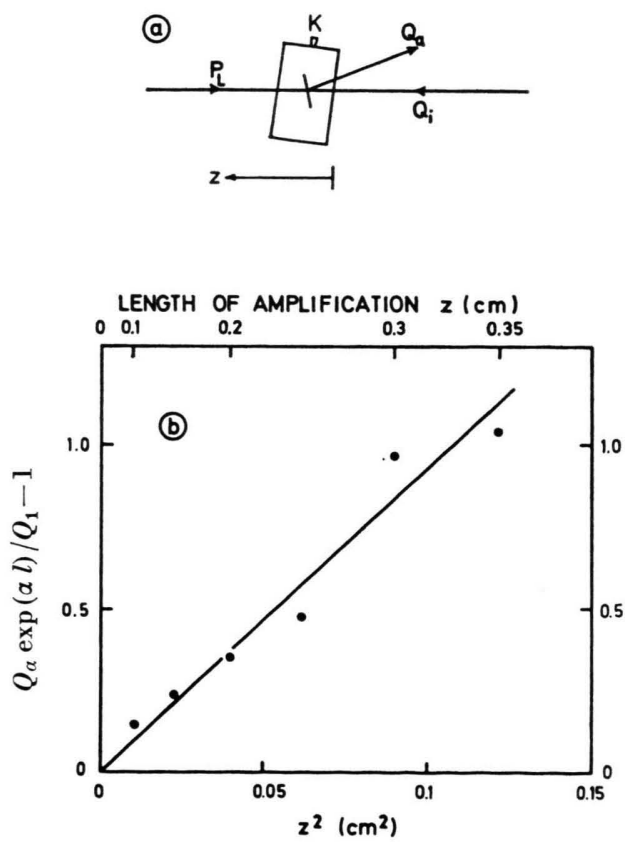

Fig. 6. a) Part of experimental system. b) Experimental am. plification (of time integrated signals) versus square of the interaction length $(\omega=0)$. 
Note the excellent agreement between our experimental values of $G$ and the calculated curve in Fig. 5.

c) It has been predicted theoretically that the amplification $G$ increases with the square of the interaction length $l$. In Fig. 6 a part of our experimental system is depicted schematically. A small fraction of the amplified input signal is coupled out by a small glass platelet at various positions within the cell. A cell of total length of $0.5 \mathrm{~cm}$ was used and the amplification was measured between $z=0.1 \mathrm{~cm}$ and $z=0.35 \mathrm{~cm}$. We worked with $\mathrm{CCl}_{4}$ and $\alpha=0.22$ $\mathrm{cm}^{-1}$. The small signal obtained in this way required the use of time integrating detectors. It is possible to show that the amplification of the time integrated signals: $Q_{\mathrm{a}} e^{\alpha z} / Q_{\mathrm{i}}-1$ is again proportional to the square of the interaction length $z$. Fig. $6 \mathrm{~b}$ depicts experimental points plotted versus $z^{2}$. The time constants of the photocell were not determined to sufficient accuracy to warrant a quantitative comparison with theory.

\section{Frequency Dependence of the Light Amplification $\left(\Theta=180^{\circ}\right)$}

In the following experiments the prism $\mathrm{Pr}$ in Fig. 2 is moved by well defined amounts in order to establish known frequency differences $\nu=\omega / 2 \pi$ between the two interacting light pulses. In Fig. 7

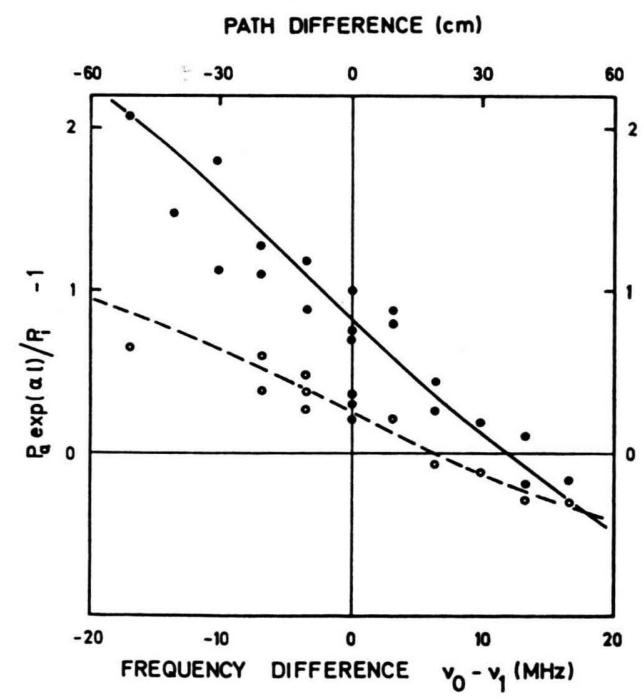

Fig. 7. Amplification at the maximum of the input pulses versus the frequency difference $\nu=v_{0}-\nu_{1}$ of the pump and signal pulse. Experimental points full circles: $\mathrm{CCl}_{4}$ with $J_{2}$ $\left(\alpha=0.09 \mathrm{~cm}^{-1}\right)$; open circles: methanol with $\mathrm{CoCl}_{2}$ $\left(\alpha=0.15 \mathrm{~cm}^{-1}\right)$. Curves are calculated. the measured amplification $G$ is plotted as a function of $v=v_{0}-v_{1}$ (lower scale) or path difference (upper scale). $G$ was determined for $\tau=0$, i. e. for the time of the peak of the pump light pulses. For large values of $v$ the amplified power is approximately three times the input power. Each point in Fig. 7 is obtained from an individual laser pulse. Two sets of measurements were made: a) $\mathrm{CCl}_{4}$ (solid circles) was investigated with $\alpha=0.09 \mathrm{~cm}^{-1}$, $\ddot{P_{0}}=0.22 \mathrm{MW}, t_{\mathrm{h}}=14 \mathrm{nsec}$ and $W=2.1 \times 10^{-2} \mathrm{~cm}$. b) The data for methanol (open circles) were the same as in a) except of $\alpha=0.15 \mathrm{~cm}$ and $\hat{P}_{0}=0.18$ MW. From these numbers $U=3.6$ and $U=1.9$ is calculated for $\mathrm{CCl}_{4}$ and methanol respectively.

The solid and broken line in Fig. 7 were calculated from Eq. (12) using theoretical values for $C_{1}(\tau=0, \omega)$ and $C_{2}(\tau=0, \omega)$ together with the experimental numbers of $U$ discussed in the preceding paragraph. It is seen from Fig. 7 that the theoretical curves acount very well for our experimental points. Theory and experiment clearly show the cross over from amplification to attenuation and the amplification at zero frequency difference $v=0$. We wish to emphasize the substantial differences to the steady state theory. The latter predicts a pronounced maximum of amplification at $\Gamma_{\mathrm{R}} / 4 \pi$, i. e. at $v=v_{0}-v_{1} \cong-9 \mathrm{MHz}$ and a symmetrical maximum of attenuation at $\nu \cong+9 \mathrm{MHz}$; in addition $G \equiv 0$ is predicted for $\nu=0$ (see Fig. 2 in I). The data presented in Fig. 7 proof again the transient nature of STRS.

\section{Angular Dependence of Light Amplification for $\omega=0$}

It has been pointed out in Section B that the Rayleigh line width $\Gamma_{\mathrm{R}}$ decreases with scattering angle $\Theta$. In liquids $t_{\mathrm{h}} \Gamma_{\mathrm{R}}<0.5$ is well satisfied for $\Theta<30^{\circ}$. As a result we obtain for $\omega=0$ and $\tau=0$ according to Eq. (9) and (10) :

$$
V_{\mathrm{R}}(\tau=0, \omega=0)=3.2 \times 10^{-2}\left(t_{\mathrm{h}} \Gamma_{\mathrm{R}} \hat{g}_{\mathrm{R}}^{\mathrm{a}} \hat{I}_{0} l\right)^{2} .
$$

Using Eqs. (3), (4), (5) and (7) it is readily shown that the right hand side of Eq. (15) is independent of angle, i. e. the amplification of infinite plane waves does not depend upon $\Theta$. The situation is markedly different for real light beams. The intensity distribution over the cross section and the interaction volume of the two intersecting light beams have to be considered. For $k>1 / W$ we use a 
Gaussian intensity distribution; the power amplification $G$ is calculated for angles $W / l<\sin \Theta / 2<0.5$

$$
G=\left(0.11 t_{\mathrm{h}} \Gamma_{\mathrm{R}} \hat{g}_{\mathrm{R}}^{\mathrm{a}} \hat{P}_{0} / \mathrm{W} \sin \Theta\right)^{2} .
$$

Equation (16) states that $G$ is proportional to $(\sin \Theta)^{-2}$, a purely geometrical factor resulting from the finite beam diameter; for increasing angles, $G$ decrease on account of the decreasing effective interaction length of the two light beams ${ }^{6}$.

We have verified Eq. (16) experimentally using the system depicted in Fig. 3 and the following experimental data: $\mathrm{CCl}_{4}$ with $\alpha=0.1 \mathrm{~cm}^{-1}, \hat{P}_{0}=0.2 \mathrm{MW}$, $W=2.1 \times 10^{-2} \mathrm{~cm}$ and $t_{\mathrm{h}}=15 \mathrm{nsec}$. The product $\Gamma_{\mathrm{R}} \hat{g_{\mathrm{R}}^{\mathrm{a}}} / \alpha=\gamma^{\mathrm{e}} \omega_{0} \beta / c n \varrho_{0} c_{p}$ is calculated from various material parameters of $\mathrm{CCl}_{4}$ to be $8.8 \times 10^{7} \mathrm{~cm}^{2}$ /MW sec [see Eqs. (5), (6) and (7)].

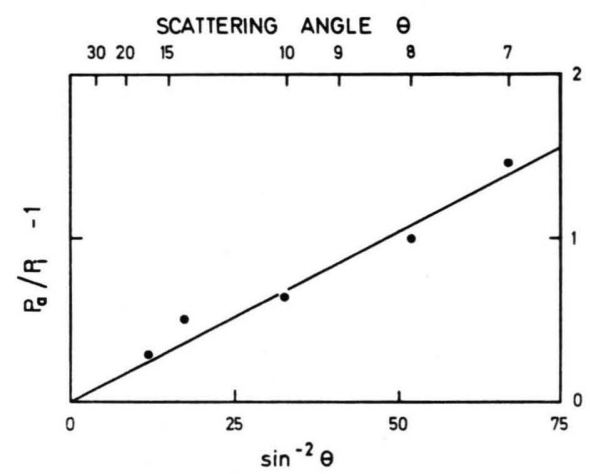

Fig. 8. Amplification at the maximum of the input pulse versus $(\sin \Theta)^{-2}$; experimental points and calculated straight line $(\omega=0)$.

In Fig. 8 the experimentally determined power amplification $G$ is plotted as a function of $(\sin \Theta)^{-2}$. At $8^{\circ}$ the power ratio $P_{\mathrm{a}} / P_{\mathrm{i}}$ is found to be two. The straight line in Fig. 8 is calculated according to Eq. (16) using the experimental data listed above. There is excellent agreement between our experimental points and the theoretical line. In this highly transient situation the pulse duration $t_{\mathrm{h}}=15 \mathrm{nsec}$ is very small compared to the time constant of the material excitation $1 / \Gamma_{\mathrm{R}}$ of several hundred nanoseconds. Experiments of the type presented in Fig. 8 allow the determination of material parameters contained in the product $\Gamma_{\mathrm{R}} \hat{g}_{\mathrm{R}}^{a}$; as expected, $\Gamma_{\mathrm{R}}$ cannot be found in this strongly transient situation.

6 W. Rother, H. Meyer, and W. Kaiser, Physics Letters 31 A, 245 [1970].

\section{E) Summary and Concluding Remarks}

In this paper a variety of experimental investigations was concerned with the light amplification via stimulated thermal Rayleigh scattering. In all experiments excellent agreement was obtained between a theory for small signal and small amplification. Of special interest was the transient amplification for $\omega=0$ i. e. for the case that the two light beams have the same frequency. The time dependence of the amplification and the quadratic dependence on the absorption coefficient was found to be in quantitative agreement with transient calculations. The frequency dependence of the amplification deviated strongly from steady state theory but was found to agree fully with transient theory. The decreasing amplification for increasing scattering angle was explained by a decreasing interaction volume of the two light beams.

For backward scattering the pulse duration $t_{\mathrm{h}}$ is approximately equal to the time constant of the material excitation $1 / \Gamma_{\mathrm{R}}$. Using amplification data at the end of the pulse (where the amplification reaches a maximum) it is possible to give an estimate of the value $\Gamma_{\mathrm{R}}$. For smaller scattering angles where $t_{\mathrm{h}}<\mathrm{l} / \Gamma_{\mathrm{R}}$ the material parameters contained in the product $\Gamma_{\mathrm{R}} \hat{g}_{\mathrm{R}}^{\mathrm{a}} / \alpha=\gamma^{\mathrm{e}} \omega_{0} \beta / c n \varrho_{0} c_{p}$ are readily determined experimentally.

For Gaussian light pulses computer calculations are necessary to make a quantitative comparison between theory and experiment. The relevant parameters $C_{1}$ and $C_{2}$ [see Eq. (9) ] have to be determined for each individual experiment. The situation is substantially simplified when the case of step functions for the pump and signal wave is considered. Analytical solutions are readily derived and comparison has been made with experimental observations. It was shown that for the first part of the pulse (i. e. for $t<1 / \Gamma_{\mathrm{R}}$ ) the experimental data can be fitted by these analytical solutions ${ }^{7}$. A time parameter $t_{0}$ had to be introduced which connected the real pulse with the time zero of the step functions. Two different experiments allowed an independent estimate of $t_{0}$. The analytical solution for $\omega=0: \quad G=(1 / 8) t^{2}\left(\hat{g}_{\mathrm{R}}^{\mathrm{a}} \Gamma_{\mathrm{R}} \hat{P}_{0} l / W_{2} \pi\right)^{2}$ is readily compared with computer calculations. At the maximum of the input pulse, we have from Eqs. (12) and (10): $G=4.3 \times 10^{-2} t_{\mathrm{h}}^{2}\left(\hat{g}_{\mathrm{R}}^{\mathrm{a}} \Gamma_{\mathrm{R}} \hat{P}_{0} l / W^{2} \pi\right)^{2}$ i. e. for $t_{\mathrm{h}}=15 \mathrm{nsec}$ we obtain $t_{0}=9 \mathrm{nsec}$ in agreement 
with the value observed in Ref. ${ }^{7}$. It should be em. phasized that the maximum in the amplification curve (for $t \cong 1 / \Gamma_{\mathrm{R}}$ ) and the decreasing amplification at the end of the pulse requires the consideration of the true pulse shape.

\section{Acknowledgement}

The authors are indebted to Drs. M. MAIER and D. PoHL for valuable discussions.

7 W. Rother, D. Pohl, and W. Kaiser, Phys. Rev. Letters 22, 915 [1969].

\title{
Charakteristische Parameter der dielektrischen Relaxationszeit starrer Dipolmolekeln in verdünnter Lösung
}

\author{
F. HUFNAGEL \\ Institut für Physik der Johannes-Gutenberg-Universität Mainz \\ (Z. Naturforsch. 25 a, 1143-1150 [1970] ; eingegangen am 17. Mai 1970) \\ Characteristic Parameters of the Dielectric Relaxation Time of Rigid Dipole Molecules \\ in Diluted Solution
}

\begin{abstract}
The dielectric relaxation time of rigid polar molecules in dilute solutions is shown to depend exponentially on an effective molecular radius which is independent of the solvent. This relation contains a temperature-dependent structure parameter, the coefficients of which are used to calculate the activation energy and -entropy of the rate process. The resulting entropies are now more satisfying due to a new formulation of the scattering factor.

Similarly the viscosity of the solvent and its structure parameters are related by an exponential law. A characteristical length in this relation is a measure of the "hole distance" of the rate process. From both exponential laws follows that the dielectric relaxation time raised to the power of the ratio of effective molecular radius to hole distance is proportional to the viscosity of the solvent.
\end{abstract}

\section{Einleitung}

Seit der Beobachtung der anomalen Dispersion polarer Molekeln im elektrischen Wechselfeld durch Drude ist man bemüht, aus der Relaxationszeit der dielektrischen Polarisation Aufschlüsse über Drehbewegungen der Molekeln in der Flüssigkeit zu erhalten. DEBYE ${ }^{1}$ versuchte zunächst mit einem einfachen hydrodynamischen Modell die Relaxationszeit der Molekel aus ihrem Volumen $V$ und der Viskosität der Flüssigkeit in erster Näherung abzuschätzen; ungeachtet ihrer besonderen Form wird die Dipolmolekel durch eine Kugel mit dem Volumen $V$ angenähert und entsprechend dem Stokesschen Gesetz die Reibungsgröße als $8 \pi \eta a^{3}$ angesetzt, so daß ihre Relaxationszeit $\tau$ durch den Ausdruck $3 \eta V / k T$ zu beschreiben ist.

Messungen an starren Dipolmolekeln in verdünnter Lösung unpolarer Lösungsmittel, also unter Aufhebung der Dipol-Dipol-Wechselwirkung, führten aber zu Relaxationszeiten, die nur etwa ein Zehntel

1 P. Debye, Polare Molekeln, Hirzel-Verlag, Leipzig 1929.

2 F. Perrin, J. Phys. 5, 497 [1934].

3 A. Budó, E. Fischer u. S. MiYamoto, Phys. Z. 40, 337 [1939]. der Debyeschen Abschätzung ausmachten. Das gilt besonders für Moleküle, deren Volumen kleiner oder gleich dem der Lösungsmoleküle ist.

Verfeinerungen des Debyeschen Ansatzes durch PERRIN ${ }^{2}$ (Ellipsoide statt Kugeln) und durch Budó, Fischer und Miyamoto ${ }^{3}$ (Einführung geeigneter Formfaktoren) ergeben in günstigen Fällen bei vergleichsweise großen Dipolmolekülen Werte, die immer noch um etwa $80 \%$ größer sind als die Meß. werte.

GIERER und WIRTZ ${ }^{4}$ versuchten dann mit diskontinuierlichen Ansätzen dem Flüssigkeitsgefüge besser Rechnung zu tragen. Ebenso erhielt HiLl ${ }^{5}$ im Rahmen ihrer Untersuchungen zur Mischviskosität zweier Flüssigkeiten Ausdrücke über die dielektrische Relaxationszeit, mit denen die Meßwerte aber nur in einzelnen Fällen verträglich sind. HASE ${ }^{6} \mathrm{er}$ weiterte mit einem Exponentialansatz die PerrinFormel, der später von NELSON ${ }^{7}$ noch vereinfacht werden konnte. Jetzt liefern diese Ausdrücke bei Dipolmolekülen, die kleiner als die umgebenden Lö-

4 A. Gierer u. K. Wirtz, Z. Naturforsch. 8 a, 532 [1953].

5 N. E. Hill, Proc. Phys. Soc. London B 67, 149 [1954].

6 H. HASE, Z. Naturforsch. 8 a, 695 [1953].

7 R. D. Nelson JR. u. C. P. Smyth, J. Phys. Amer. 68, 2704 [1964]. 